Original

\title{
Riesgo de mortalidad total y cardiovascular según la clasificación propuesta por las guías KDIGO en pacientes con diabetes tipo 2
}

\author{
Ana Lidia Medrano Navarro ${ }^{\mathrm{a}, *}$, Alicia Justel Enríquez ${ }^{\mathrm{b}}$, Javier Alameda Serrano ${ }^{\mathrm{c}}$, \\ Yolanda Blasco Lamarca ${ }^{\mathrm{d}}$, Daniel Sáenz Abad ${ }^{\mathrm{e}} \mathrm{y}$ José Antonio Gimeno Orna ${ }^{\mathrm{d}, \mathrm{e}}$ \\ a Unidad de Endocrinología y Nutrición, Hospital de Barbastro, Barbastro, Huesca, España \\ b Servicio de Endocrinología y Nutrición, Hospital de Huelva, Huelva, España \\ c Servicio de Cardiología, Hospital Royo Villanova, Zaragoza, España \\ d Servicio de Endocrinología y Nutrición, Hospital Clínico Lozano Blesa, Zaragoza, España \\ e Instituto de Investigación Sanitaria de Aragón, Hospital Clínico Universitario Lozano Blesa, Zaragoza, España
}

\section{INFORMACIÓN DEL ARTÍCULO}

\section{Historia del artículo:}

Recibido el 21 de junio de 2018

Aceptado el 10 de enero de 2019

On-line el xxx

\section{Palabras clave:}

Diabetes mellitus

Supervivencia

Pronóstico

\section{R E S U M E N}

Antecedentes y objetivo: El objetivo del estudio fue comprobar la validez de la clasificación de riesgo KDIGO 2012 para predecir mortalidad total (MT) y cardiovascular (MCV) en diabetes mellitus tipo 2 (DM2). Materiales y métodos: Estudio de cohortes prospectivo incluyendo pacientes con DM2. Los puntos finales clínicos fueron MT y MCV. La principal variable predictora fue la clasificación KDIGO, una variable que recoge 4 niveles de riesgo en dependencia de una combinación de la tasa de filtración glomerular y la excreción de albúmina urinaria. La evaluación del poder predictivo se realizó con el índice de mejora de discriminación integrada (IDI).

Resultados: Se incluyeron 453 pacientes (39,3\% varones, edad 64,9 [DE 9,3] años y evolución de DM2 de 10,4 [DE 7,5] años). Durante una mediana de 13 años de seguimiento, hubo incremento significativo de la tasa/1000 pacientes-año de MT (26,5 vs. 45,1 vs. 79,2 vs. 109,8 ; p<0,001) y de MCV ( 8,1 vs. 17,4 vs. 24,7 vs. 57,$5 ;$ p <0,001) en las sucesivas categorías de riesgo KDIGO. En análisis multivariante también hubo incremento de riesgo de MT (HR[riesgo moderado]=1,29; HR[riesgo alto] = 1,83; HR[riesgo muy alto]=2,15; $\mathrm{p}=0,016)$ y MCV (HR[riesgo moderado]=1,73; HR[riesgo alto] =2,27; HR[riesgo muy alto] $=4,22 ; \mathrm{p}=0,007$ ) en las sucesivas categorías. La clasificación KDIGO mejoró la predicción de MT (IDI =0,00888; $\mathrm{p}=0,047$ ) y MCV (IDI =0,01813; $\mathrm{p}=0,035)$.

Conclusiones: La clasificación de riesgo según guías KDIGO 2012 puede estratificar eficazmente el riesgo de MT y MCV en pacientes con DM2.

(C) 2019 Elsevier España, S.L.U. Todos los derechos reservados.

\section{Total and cardiovascular mortality risk according to KDIGO guidelines classification in type 2 diabetic patients}

\section{A B S T R A C T}

Background and aims: Our aim was to assess the usefulness of KDIGO 2012 risk classification to predict total and cardiovascular mortality in type 2 diabetes mellitus (DM2).

Material and methods: Prospective cohort study that included DM2 patients. Clinical end-points were total and cardiovascular mortality. The main predictive variable was KDIGO risk classification, which is a combination of urinary albumin excretion and glomerular filtration rate. The predictive value was evaluated by the integrated discrimination improvement (IDI) index.

Results: 453 patients (39.3\% males, aged 64.9 [SD 9.3] and with a mean diabetes duration of 10.4 [SD 7.5] years) were included. During a median follow-up of 13 years, mortality rates per 1000 patients/year (26.5 vs. 45.1 vs. 79,2 vs. 109,$8 ; p<0,001$ ) and cardiovascular mortality (8.1 vs. 17.4 vs. 24.7 vs. 57.5 ; $\mathrm{p}<0,001)$ were progressively increased in successive KDIGO categories. In the multivariate analysis, there

\footnotetext{
* Autor para correspondencia.

Correo electrónico: analidia.medrano@gmail.com (A.L. Medrano Navarro).
} 
was also a progressive increase of mortality risk $(\mathrm{HR}[$ moderate risk] $=1.29$; HR[high risk]) $=1.83$; $\mathrm{HR}[$ very high risk] $=2.15 ; \mathrm{p}=.016$ ) and cardiovascular mortality risk (HR[moderate risk] = 1.73; HR[high risk] $=2.27$; HR[very high risk] $=4.22 ; \mathrm{p}=.007$ ) in the successive categories. KDIGO classification was able to improve the mortality risk prediction (IDI $=0.00888 ; \mathrm{p}=.047)$ and cardiovascular mortality risk prediction (IDI $=0.01813 ; \mathrm{p}=.035$ ).

Conclusions: KDIGO risk classification can effectively stratify total and cardiovascular mortality risk in DM2 patients.

(c) 2019 Elsevier España, S.L.U. All rights reserved.

\section{Introducción}

La presencia de diabetes mellitus (DM) incrementa el riesgo cardiovascular de los pacientes, y es considerada un equivalente de riesgo coronario ${ }^{1}$. A pesar de ello el riesgo puede diferir ampliamente entre unos pacientes y otros, incluso entre aquellos con enfermedad cardiovascular (ECV) inicial; en el ensayo IMPROVE-IT los pacientes con afectación de más de un territorio vascular tenían un riesgo de mortalidad cardiovascular de un 30\% extrapolado a 10 años $^{2}$.

Es ampliamente reconocido que la presencia de enfermedad renal crónica (ERC) incrementa el riesgo de mortalidad total (MT) y mortalidad cardiovascular (MCV) en población general: el aumento de riesgo se produce tanto por la elevación de la excreción de albúmina urinaria (EAU) como por la reducción de la tasa de filtración glomerular (TFG), proporcionando ambas variables una información aditiva ( $\sin$ interacción) e independiente ${ }^{3}$.

Los pacientes con nefropatía diabética tienen tasas de MT y MCV elevadas ${ }^{4}$ que pueden sobrepasar ampliamente el umbral del muy alto riesgo (riesgo de MCV superior al 10\% en 10 años) según la definición de las guías de la European Society of Cardiology 5 . Los pacientes con DM tipo 2 y ECV establecida son considerados en algunas guías de práctica clínica como de riesgo extremo ${ }^{6}$, aunque no existe un umbral consensuado que defina este riesgo extremo.

Las guías de 2012 del Kidney Disease Improving Global Outcomes (KDIGO) ${ }^{7}$, definen 4 categorías de riesgo para el pronóstico combinado de MT, MCV y sucesos relacionados con la reagudización y progresión de la ERC. Se reconoce además que el abordaje del paciente con DM y ERC, enfocado a reducir su elevado riesgo cardiovascular, debe ser multidisciplinar, con implicación de especialidades entre las que destacan, además de medicina interna y endocrinología, cardiología y nefrología.

Los objetivos de nuestro trabajo, mediante el seguimiento a largo plazo de una cohorte de pacientes con DM tipo 2, fueron:

1. Evaluar si la clasificación de riesgo combinada propuesta por KDIGO recoge la misma información que la suma de la TFG y la EAU.

2. Comprobar la validez de la clasificación en 4 categorías, definidas por las guías KDIGO, para predecir MT y MCV en pacientes con DM tipo 2.

\section{Material y métodos}

Diseño: estudio de cohortes prospectivo

Población a estudio. Está descrita detalladamente en artículos previos ${ }^{8,9}$. Entre el 1 de junio de 1994 y el 1 de junio de 1998 se seleccionaron 463 pacientes con DM tipo 2 atendidos en consultas externas de endocrinología del Hospital Comarcal de Alcañiz. Dicho hospital tiene una población de referencia de 70000 personas.
Los criterios de inclusión fueron: diabetes diagnosticada por los criterios de la Organización Mundial de la Salud vigentes en ese momento ${ }^{10}$, edad superior a 35 años en el momento del diagnóstico de la DM y ausencia de tratamiento con insulina al menos durante un año tras el diagnóstico de la enfermedad. Los criterios de exclusión fueron enfermedad somática grave, gestación, situación de hipertiroidismo o hipotiroidismo no controlados, tratamiento con corticoides sistémicos e insuficiencia renal avanzada (creatinina superior a $3 \mathrm{mg} / \mathrm{dl}$ ). El diseño del estudio fue aprobado por el hospital y todos los pacientes dieron su consentimiento verbal antes de ser incluidos en el mismo.

Examen basal. Se realizó una entrevista en el momento de la inclusión, con anotación de edad, sexo, tiempo de evolución conocido de la enfermedad, hábito tabáquico (fumadores activos, exfumadores y no fumadores), tratamiento de la DM (dieta, hipoglucemiantes orales o insulina) y cualquier manifestación de enfermedad vascular prevalente (cardiopatía isquémica, isquemia de extremidades inferiores y/o enfermedad cerebrovascular). En el examen físico se determinaron peso y talla (con cálculo del índice de masa corporal, definido como peso en $\mathrm{kg} /$ talla al cuadrado en metros), presión arterial sistólica y diastólica, con clasificación de los pacientes en hipertensos si la presión sistólica era superior a $140 \mathrm{mmHg}$ y/o la diastólica superior a $90 \mathrm{mmHg}$ o seguían tratamiento con hipotensores. El fondo de ojo se visualizó mediante oftalmoscopia directa e indirecta y biomicroscopia con lentes de 78 y/o 90 Dp tras midriasis con tropicamida y, en determinados casos, fenilefrina. Los hallazgos se clasificaron de la siguiente forma: 1) sin signos de retinopatía diabética, 2) retinopatía simple o de fondo ( 2 o más microaneurismas en ambos ojos) y 3) retinopatía proliferativa (aparición de nuevos vasos, presencia de hemorragia vítrea o presencia de cicatrices de fotocoagulación dirigidas al tratamiento de nuevos vasos).

Se realizó extracción de muestra de sangre venosa, tras ayuno nocturno de $10 \mathrm{~h}$ y antes de la administración de la medicación antidiabética, con determinación de glucemia, hemoglobina glicada (HbA1c), creatinina, colesterol total y triglicéridos. Se recogió una muestra de orina de $24 \mathrm{~h} \mathrm{y}$, tras excluir infección urinaria, se determinó la tasa de excreción de albúmina urinaria, con clasificación de las muestras en normoalbuminúricas (<30 mg), microalbuminúricas (30-300 mg) y macroalbuminúricas o proteinúricas (>300 mg). Las mediciones bioquímicas se realizaron mediante autoanalizador Shimadzu CL 7200. La HbA1c se midió en analizador automático Cobas Mira Plus, con un rango de normalidad entre 4,5 y 5,7\%. La EAU se determinó con un método inmunoturbidimétrico en analizador Cobas Integra 700.

Se calculó la TFG en $\mathrm{ml} / \mathrm{min}$ por $1,73 \mathrm{~m}^{2}$ mediante la fórmula de CKD-EPI:

$\mathrm{Si}$ (mujer y creatina < $=0,7) \mathrm{TFG}=144^{*}\left([\text { creatina } / 0,7]^{* *}-0,329\right)$ $*\left(0,993{ }^{* *}\right.$ edad $)$.

Si (mujer y creatina $>0,7) \mathrm{TFG}=144^{*}\left([\text { creatina } / 0,7]^{* *}-1.209\right)^{*}$ $\left(0,993^{* *}\right.$ edad $)$.

Si (varón y creatina $<=0,9$ ) TFG $=141 *$ ([creatina / 0,9] ${ }^{* *}$ $0,411)^{*}\left(0,993^{* *}\right.$ edad $)$. 
$\mathrm{Si}($ varón y creatina $>0,9) \mathrm{TFG}=141^{*}\left([\text { creatina } / 0,9]^{* *}-1.209\right)^{*}$ $(0,993$ ** edad).

Seguimiento de la cohorte. Se siguieron de modo prospectivo todos los pacientes hasta su fallecimiento o hasta la fecha de cierre del estudio el 31 de agosto de 2012. Las causas de muerte se obtuvieron a partir de la historia clínica hospitalaria o mediante contacto con el médico de atención primaria que firmó el certificado de defunción. Se consideraron como cardiovasculares las muertes súbitas, las debidas a infarto de miocardio o insuficiencia cardiaca terminal y las debidas a enfermedad cerebrovascular. Se definió como infarto de miocardio la constatación en la historia clínica de ingreso por dolor torácico con cambios electrocardiográficos típicos y/o elevación característica y descenso progresivo de las cifras de CPK-MB y/o troponina I (disponible esta última desde febrero del año 2000 en el hospital). Un accidente cerebrovascular fue definido como un déficit neurológico que persistía más de $24 \mathrm{~h}$. En 4 pacientes $(0,9 \%)$ no se pudo determinar su estatus vital y fueron considerados pérdidas en el seguimiento.

Métodos estadísticos. Las variables cuantitativas se describen con su media y desviación estándar (DE) y las cualitativas con distribución de frecuencias. Las variables cuantitativas se comparan con ANOVA o prueba no paramétrica de Kruskal-Wallis (tiempo de evolución de la DM y triglicéridos). Para la comparación de variables cualitativas se utilizó test de $\mathrm{X}^{2}$ con evaluación de tendencia lineal.

Como variables primarias de resultado se consideraron la MT y la MCV. Como variables predictoras se analizaron:

1. TFG codificada en $3(\geq 60 ; 45-59 ;<45)$ categorías según la TFG expresada en $\mathrm{ml} / \mathrm{min}$ por $1,73 \mathrm{~m}^{2}$.

2. EAU dividida en $3(<30 ; 30-300 ;>300)$ categorías según el valor en mg registrado en la recogida de orina de $24 \mathrm{~h}$.

3. Clasificación de riesgo KDIGO codificada en 4 categorías:

- Riesgo bajo: TFG $\geq 60$ y EAU $<30$.

- Riesgo moderado: TFG $\geq 60$ y EAU $30-300$ o TFG $45-59$ y EAU $<30$.

- Riesgo alto: TFG $\geq 60$ y EAU >300 o TFG 45-59 y EAU 30-300 o TFG $30-44$ y EAU $<30$.

- Riesgo muy alto: TFG 45-59 y EAU >300 o TFG 30-44 y EAU $\geq 30$ o TFG $<30$.

Se realizó seguimiento de los pacientes desde su inclusión hasta su salida del estudio por alcanzar la fecha de cierre del mismo, por muerte o por pérdida. Las tasas de incidencia (TI) de los distintos episodios se expresan por cada 1000 pacientes-año. La relación entre la TI y el riesgo $(R)$ a 10 años puede establecerse fácilmente con la fórmula: $\mathrm{R}=1-\left(\mathrm{e}^{-\mathrm{TI}{ }^{*} d u r a c i o ́ n}\right)$. En nuestro trabajo consideramos como extremo un R de MCV similar al de los pacientes con ECV inicial (superior al 20\% en 10 años). La comparación de tasas entre las diferentes categorías consideradas se realizó mediante análisis de Kaplan-Meier y log rank test.

Posteriormente se realizaron modelos de regresión de Cox, con estimación de las hazard ratios (HR) y sus intervalos de confianza (IC) del 95\%, para evaluar el riesgo que confiere la presencia de las distintas categorías de TFG, EAU y riesgo KDIGO. Se realizaron modelos univariante y multivariante con ajuste para edad, sexo, tabaquismo, presencia de hipertensión arterial y enfermedad vascular prevalente, colesterol y triglicéridos (introducido en forma logarítmica), HbA1c y tiempo de evolución de la DM. Se realizó adicionalmente ajuste para presencia y grado de retinopatía, ya que dicha complicación microangiopática guarda una estrecha relación con el tiempo de evolución de la enfermedad y ha demostrado en nuestra cohorte ser un factor predictivo de mortalidad ${ }^{9}$. Mediante procedimiento de exclusión secuencial se determinaron los predictores independientes de MT y MCV.

Sobre un modelo base (compuesto por edad, sexo, tabaquismo, presencia de hipertensión arterial y enfermedad vascular prevalente, colesterol, triglicéridos, HbA1c, tiempo de evolución de la
DM y presencia de retinopatía) se evaluaron 2 modelos completos: modelo 1 (modelo base + clasificación KDIGO) y modelo 2 (modelo base + variables categóricas TFG y EAU). La diferencia en el poder de predicción de los modelos 1 y 2 se determinó mediante el estadístico C de Harrell. La utilidad predictiva para MT y MCV de la clasificación KDIGO se evaluó mediante el cálculo del índice Integrated Discrimination Improvement (IDI). Se efectuó prueba de Hosmer y Lemeshow para comprobar la calibración de los modelos (un valor de p no significativo indica un ajuste adecuado entre los sucesos observados y los pronosticados a partir del modelo).

Se incluyó un término de interacción entre el riesgo KDIGO y la presencia de enfermedad vascular prevalente, y posteriormente se realizó análisis estratificado en dependencia de la presencia o ausencia inicial de enfermedad vascular. Considerando como una prioridad identificar entre los pacientes con diabetes tipo $2 \sin \mathrm{ECV}$ prevalente aquellos con un riesgo cardiovascular extremo (y similar al de los sujetos con ECV inicial establecida), se evaluó el Net Reclassification Improvement entre el modelo base y el modelo 1 para reclasificar pacientes con un punto de corte en R de MCV superior al $20 \%$ en 10 años.

Las asociaciones con $\mathrm{p}<0,05$ fueron consideradas significativas. Se utilizó como programa informático SPSS versión 22.0.

\section{Resultados}

Se incluyeron 453 de los 463 pacientes debido a que en 10 de ellos no se tenía información sobre la TFG y/o la EAU. Entre los pacientes incluidos hubo 178 (39,3\%) varones, la edad media fue de 64,9 (DE 9,3) años y el tiempo de evolución medio de la DM de 10,4 (DE 7,5) años. En el momento de la inclusión 192 (42,4\%) pacientes llevaban tratamiento con insulina y 98 (21,6\%) tenían enfermedad vascular prevalente.

El 30,2\% de los pacientes tenían una TFG $<60 \mathrm{ml} / \mathrm{min}$ por 1,73 $\mathrm{m}^{2}$, mientras que las respectivas prevalencias de normo-, micro- $\mathrm{y}$ macroalbuminuria fueron de 70,9, 23,1 y 6\%. La distribución de los pacientes según las categorías combinadas de TFG y EAU se presenta en la tabla 1 . El riesgo KDIGO fue bajo en $51,7 \%$, moderado en $32,7 \%$, alto en $10,2 \%$ y muy alto en $5,5 \%$.

Las características de los pacientes en dependencia de su riesgo KDIGO quedan reflejadas en la tabla 2. A medida que se avanzaba en la categoría de riesgo KDIGO los pacientes tenían por definición menor TFG y mayor EAU; además presentaban mayor edad, HbA1cy tiempo de evolución de la DM. También eran mayores las prevalencias de hipertensión arterial, enfermedad vascular (sobre todo por isquemia de extremidades inferiores), retinopatía y tratamiento insulínico iniciales.

Durante una mediana de 13 años de seguimiento se produjeron 207 muertes totales (tasa 39,5/1000) y 74 muertes cardiovasculares (tasa 14,1/1000). Como era de esperar, la tasa de MCV fue superior en sujetos con ECV inicial que en sujetos sin ella ( 27,3 vs. $11,0 / 1000$; $\mathrm{p}<0,001)$. Las tasas respectivas de MT y MCV en dependencia de la TFG, la EAU y la clasificación de riesgo KDIGO quedan reflejadas en la tabla 3. Tanto para MT como para MCV hubo una tendencia lineal significativa en el incremento progresivo de tasas a medida que se deterioraban la TFG y la EAU, así como desde los grupos de bajo a muy alto riesgo según clasificación KDIGO ( $\mathrm{p}<0,001$ todas ellas). Las categorías de riesgo KDIGO alto y muy alto superaron el riesgo de MCV del 20\% en 10 años ( $R$ del 22\% en pacientes con KDIGO de alto riesgo y $\mathrm{R}$ del $44 \%$ en pacientes con KDIGO de muy alto riesgo) al igual que ocurría con los pacientes con ECV inicial ( $R$ del 24\%). Las curvas de supervivencia según el riesgo KDIGO están plasmadas en la figura 1 (mortalidad total) y figura 2 (mortalidad cardiovascular).

La clasificación de riesgo KDIGO estuvo asociada con un incremento del riesgo de MT y MCV tanto en análisis univariante como en análisis multivariante (tabla 4) con ajuste completo (edad, 
Tabla 1

Porcentaje de pacientes en cada categoría combinada de TFG y EAU

\begin{tabular}{|c|c|c|c|}
\hline \multirow[b]{2}{*}{ Tasa de filtración glomerular (ml/min por $\left.1,73 \mathrm{~m}^{2}\right)$} & \multicolumn{3}{|c|}{ Excreción albúmina urinaria (mg/24h) } \\
\hline & $<30(\%)$ & $30-300(\%)$ & $>300(\%)$ \\
\hline$\geq 60(\%)$ & 51,7 & 15,7 & 2,4 \\
\hline $45-59(\%)$ & 17 & 5,5 & 0,9 \\
\hline $30-44(\%)$ & 2,2 & 1,8 & 2 \\
\hline$<30(\%)$ & 0 & 0,2 & 0,7 \\
\hline
\end{tabular}

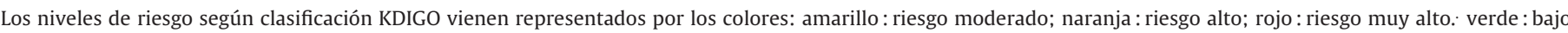
riesgo. Los colores de la tabla solo pueden apreciarse en la versión electrónica del artículo.

Tabla 2

Características de los pacientes según clasificación de riesgo KDIGO en el momento de la inclusión

\begin{tabular}{|c|c|c|c|c|c|}
\hline Riesgo KDIGO & Bajo & Medio & Alto & Muy alto & $\mathrm{p}$ \\
\hline TFG $\left(\mathrm{ml} / \mathrm{min}\right.$ por $\left.1,73 \mathrm{~m}^{2}\right)$ & $73,9(9,9)$ & $63,5(10,6)$ & $56,2(11,6)$ & $40,6(10,2)$ & $<0,001$ \\
\hline Microalbuminuria (\%) & 0 & 48 & 54,3 & 36 & $<0,001$ \\
\hline Macroalbuminuria (\%) & 0 & 0 & 23,9 & 64 & \\
\hline Edad (años) & $62(9,1)$ & $67,2(8,6)$ & $68,8(8,2)$ & $71,8(7,4)$ & $<0,001$ \\
\hline Sexo (\% varones) & 43,6 & 32,4 & 43,5 & 32 & ns \\
\hline ECV prevalente (\%) & 16,7 & 20,3 & 41,3 & 40 & $<0,001$ \\
\hline Cardiopatía isquémica (\%) & 9,4 & 10,1 & 13 & 16 & ns \\
\hline $\mathrm{ACV}(\%)$ & 5,1 & 6,1 & 15,2 & 12 & 0,025 \\
\hline Isquemia EEII (\%) & 3 & 6,8 & 26,1 & 24 & $<0,001$ \\
\hline Tabaco (\% activo) & 14,1 & 8,1 & 14,3 & 4 & ns \\
\hline HTA (\%) & 70,5 & 82,4 & 91,3 & 96 & $<0,001$ \\
\hline Colesterol (mg/dl) & $223(41)$ & $214(38)$ & $212(41)$ & $225(45)$ & ns \\
\hline Triglicéridos (mg/dl) & 133(87) & 135(93) & $150(126)$ & $156(57)$ & ns \\
\hline $\operatorname{IMC}\left(\mathrm{kg} / \mathrm{m}^{2}\right)$ & $29,1(4,7)$ & $29,2(4,4)$ & $30,4(5,6)$ & $30,1(4,4)$ & ns \\
\hline HbA1c (\%) & $7,6(1,5)$ & $7,8(1,6)$ & $8,3(1,9)$ & $8(1,4)$ & 0,048 \\
\hline Evolución DM (años) & $8,7(6,9)$ & $11,9(7,9)$ & $12,9(7,6)$ & $13,2(7,7)$ & $<0,001$ \\
\hline Tratamiento insulina (\%) & 32,2 & 47,3 & 65,2 & 68 & $<0,001$ \\
\hline Retinopatìa simple & 25,1 & 24,5 & 45,7 & 29,2 & $<0,001$ \\
\hline Retinopatía proliferativa (\%) & 5,2 & 12,2 & 15,2 & 37,5 & \\
\hline
\end{tabular}

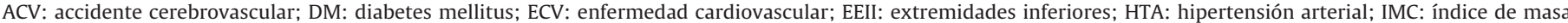
corporal; TFG: tasa de filtración glomerular.

Tabla 3

Tasas de mortalidad total y cardiovascular según características iniciales de los pacientes. Comparación de tasas mediante log rank test

\begin{tabular}{|c|c|c|c|c|}
\hline Tasas/1000 pacientes-año & MT & $\mathrm{p}$ & MCV & $\mathrm{p}$ \\
\hline \multicolumn{5}{|l|}{ Clasificación según TFG } \\
\hline $\mathrm{TFG} \geq 60$ & 30,3 & $<0,001$ & 10,8 & $<0,001$ \\
\hline TFG 45-59 & 57 & & 18,1 & \\
\hline TFG $<45$ & 110,6 & & 51,1 & \\
\hline \multicolumn{5}{|l|}{ Clasificación según EAU } \\
\hline $\mathrm{EAU}<30$ & 33,6 & $<0,001$ & 10,5 & $<0,001$ \\
\hline EAU $30-300$ & 48 & & 18,6 & \\
\hline EAU $>300$ & 104,2 & & 56,8 & \\
\hline \multicolumn{5}{|c|}{ Clasificación según riesgo KDIGO } \\
\hline R-KDIGO bajo & 26,5 & $<0,001$ & 8,1 & $<0,001$ \\
\hline R-KDIGO medio & 45,1 & & 17,4 & \\
\hline R-KDIGO alto & 79,2 & & 24,7 & \\
\hline R-KDIGO muy alto & 109,8 & & 57,5 & \\
\hline
\end{tabular}

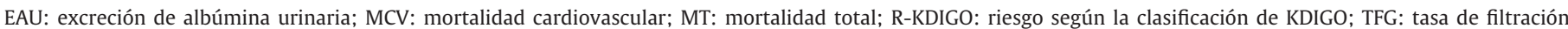
glomerular.

sexo, tabaquismo, presencia de hipertensión arterial y enfermedad vascular, colesterol y triglicéridos, HbA1c, tiempo de evolución de la DM y presencia y grado de retinopatía). El mejor modelo seleccionado para predecir MT estuvo compuesto por la clasificación riesgo KDIGO (HR[riesgo moderado]=1,29; IC 95\% 0,9-1,8; $\mathrm{HR}$ [riesgo alto]=1,83; IC 95\% 1,16-2,9; HR[riesgo muy alto] =2,15; IC 95\% 1,2-3,7; $\mathrm{p}=0,016)$, la edad (HR[1 año] =1,09; IC 95\% 1,07$1,11 ; \mathrm{p}<0,0001)$, la presencia de retinopatía simple $(H R=1,42$; IC $95 \% 1-2 ; p=0,049)$ o proliferativa ( $\mathrm{HR}=2,27$; IC 95\% 1,4-3,6; $\mathrm{p}<0,001$ ), el tabaquismo activo ( $\mathrm{HR}=1,87$; IC 95\% $1,2-3 ; \mathrm{p}=0,007$ ) y la presencia de enfermedad vascular inicial ( $H R=1,71$; IC $95 \%$ $1,2-2,4 ; \mathrm{p}=0,002)$. El mejor modelo seleccionado para predecir MCV estuvo compuesto por la clasificación de riesgo KDIGO
(HR[riesgo moderado] = 1,73; IC 95\% 1-3,1; HR[riesgo alto] = 2,27; IC 95\% 1,03-5; HR[riesgo muy alto] = 4,22; IC 95\% 1,8-9,6; p=0,007), la edad (HR[1 año]=1,08; IC 95\% 1,04-1,11; p<0,0001), y la presencia de enfermedad vascular inicial ( $H R=2,27$; IC 95\% 1,3-3,9; $p$ $=0,004)$.

Para MT, el valor del estadístico C de Harrell fue de 0,744 en el modelo de base, 0,751 en el modelo 1 y 0,751 en el modelo 2 ( $\mathrm{p}=\mathrm{ns}$ modelo 1 vs. modelo 2). El IDI del modelo 1 respecto al modelo de base fue de 0,00888 ( $\mathrm{p}=0,047$ ).

Para MCV, el valor del estadístico C de Harrell fue de 0,743 en el modelo de base, 0,770 en el modelo 1 y 0,762 en el modelo 2 ( $\mathrm{p}=\mathrm{ns}$ modelo 1 vs. modelo 2). El IDI del modelo 1 respecto al modelo de base fue de $0,01813(\mathrm{p}=0,035)$. 


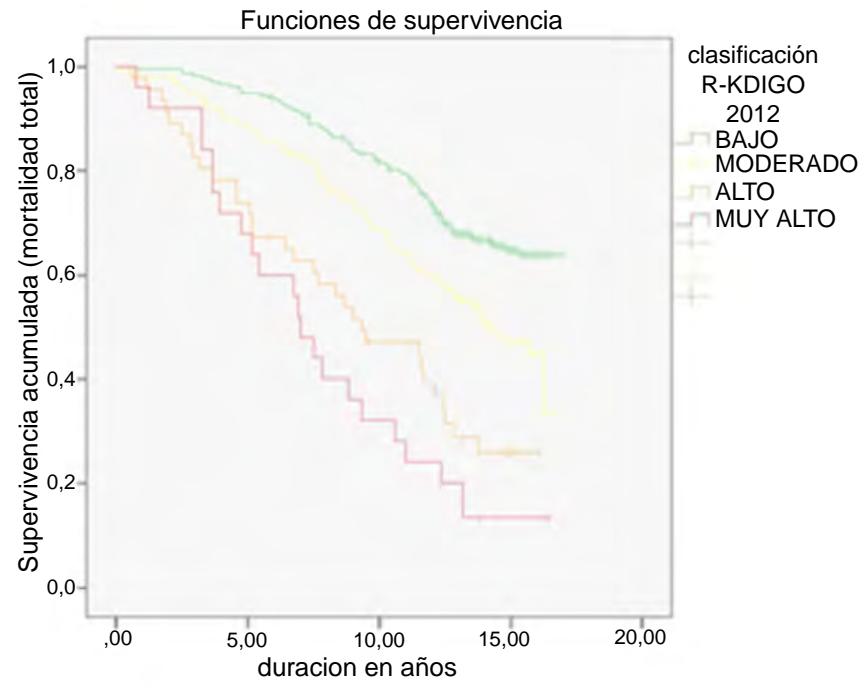

Figura 1. Probabilidad estimada mediante curvas de Kaplan-Meier de supervivencia en dependencia del nivel de riesgo según la clasificación KDIGO ( $p<0,0001)$.

El valor de la significación estadística de la prueba de Hosmer y Lemeshow en el modelo 1 de MT fue de $\mathrm{p}=0,290 \mathrm{y}$ en el modelo 1 de MCV fue de $\mathrm{p}=0,357$ indicando una calibración adecuada de los modelos que incluían la clasificación de riesgo KDIGO.

El término de interacción entre clasificación de riesgo KDIGO y la presencia de enfermedad vascular inicial no fue significativo para $\operatorname{MCV}(p=0,13)$, pero quedó en el límite de la significación $(\mathrm{p}=0,063)$ para $\mathrm{MT}$, por lo que se realizó análisis estratificado sobre el pronóstico de MT según la presencia o no de enfermedad vascular prevalente. En pacientes sin macroangiopatía la clasificación de riesgo KDIGO (HR[riesgo moderado] = 1,18; IC 95\% 0,8-1,7; $\mathrm{HR}$ [riesgo alto]=1,74; IC 95\% 1-3,1; HR[riesgo muy alto]=5,24; IC 95\% 2,7-10,2; $\mathrm{p}<0,001)$ tuvo mayor poder predictivo que en pacientes con macroangiopatía (HR[riesgo moderado] $=1,44$; IC 95\% 0,7-2,8; HR[riesgo alto] = 2,27; IC 95\% 1,1-4,7; HR[riesgo muy alto] = 0,9; IC 95\% 0,4-2,2; $\mathrm{p}=0,11$ ).

En sujetos sin enfermedad vascular inicial, el Net Reclassification Improvement al aplicar el modelo predictivo para MCV con inclusión de la clasificación KDIGO permitió reclasificar correctamente al $11,96 \%(p=0,055)$ de los pacientes. Entre los pacientes con episodio de MCV subieron de categoría de riesgo 6 y bajó 1 (mejora del

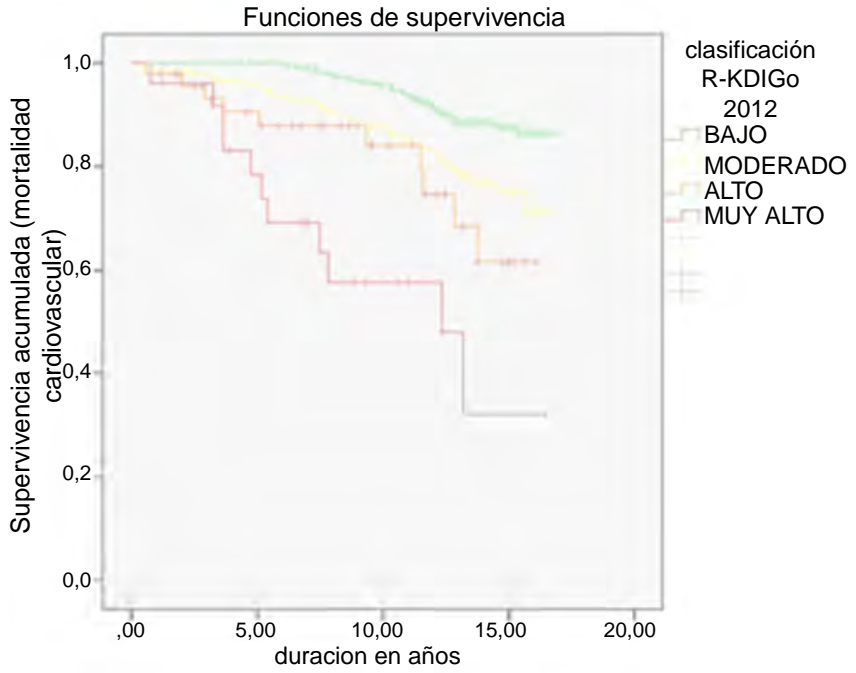

Figura 2. Probabilidad estimada mediante curvas de Kaplan-Meier de supervivencia sin experimentar muerte de origen cardiovascular en dependencia del nivel de riesgo según la clasificación KDIGO ( $p<0,0001$ ).

$11,63 \% ; p=0,059)$ y entre los pacientes sin episodio de MCV bajaron de categoría de riesgo 5 y subieron 4 (mejora del $0,33 \%$; $\mathrm{p}=0,74$ ).

\section{Discusión}

Mediante un seguimiento prospectivo durante una mediana de 13 años de una cohorte de 453 pacientes con DM tipo 2, hemos podido comprobar la validez de la clasificación de riesgo en 4 categorías, propuesta por las guías KDIGO en 2012, para predecir el riesgo de mortalidad total y mortalidad cardiovascular. La clasificación KDIGO aportaba una información pronóstica similar a la aportada por la consideración conjunta de la TFG y la EAU y demostró una mejora significativa del índice IDI. Además permitió reclasificar al 11,6\% de los pacientes sin ECV inicial a un riesgo similar al de los pacientes con ECV establecida.

El tema de la consideración de la diabetes como un equivalente de riesgo coronario ha sido ampliamente debatido. En el clásico estudio de Haffner et al. ${ }^{1}$ los pacientes con DM tipo 2 sin infarto de miocardio previo tenían un riesgo vascular similar al de pacientes sin DM pero que habían experimentado un infarto. Un seguimiento

Tabla 4

Riesgo de mortalidad total y cardiovascular en dependencia de las características iniciales de los pacientes

\begin{tabular}{|c|c|c|c|c|c|c|c|c|}
\hline & \multicolumn{2}{|c|}{ MT univariante } & \multicolumn{2}{|c|}{ MT ajustada } & \multicolumn{2}{|c|}{ MCV univariante } & \multicolumn{2}{|c|}{ ajustada } \\
\hline & HR & IC $95 \%$ & HR & IC $95 \%$ & HR & IC 95\% & HR & IC $95 \%$ \\
\hline \multicolumn{9}{|c|}{ Clasificación según tasa de filtración glomerular } \\
\hline $\mathrm{TFG} \geq 60$ & Referencia & & Referencia & & Referencia & & Referencia & \\
\hline TFG 45-59 & 1,96 & $1,4-2,7$ & 1,29 & $0,9-1,8$ & 1,80 & $1,1-3,1$ & 1,38 & $0,7-2,5$ \\
\hline TFG $<45$ & 4,44 & $2,9-6,8$ & 1,62 & $0,9-2,8$ & 6,00 & $3,1-11$ & 1,59 & $0,7-3,8$ \\
\hline \multicolumn{9}{|c|}{ Clasificación según excreción de albúmina urinaria } \\
\hline $\mathrm{EAU}<30$ & Referencia & & Referencia & & Referencia & & Referencia & \\
\hline EAU $30-300$ & 1,49 & $1,1-2$ & 0,92 & $0,6-1,3$ & 1,87 & $1,1-3,2$ & 1,19 & $0,6-2,2$ \\
\hline $\mathrm{EAU}>300$ & 3,62 & $2,3-5,7$ & 2,41 & $1,3-4,5$ & 6,52 & $3,4-12$ & 4,43 & $1,7-11,3$ \\
\hline \multicolumn{9}{|c|}{ Clasificación DE R- KDIGO } \\
\hline Bajo & Referencia & & Referencia & & Referencia & & Referencia & \\
\hline Medio & 1,75 & $1,3-2,4$ & 1,24 & $0,9-1,8$ & 2,26 & $1,3-3,9$ & 1,91 & $1-3,5$ \\
\hline Alto & 3,40 & $2,2-5,1$ & 1,77 & $1,1-2,8$ & 3,63 & $1,7-7,6$ & 2,21 & $1-5,1$ \\
\hline Muy alto & 5,10 & $3,1-8,3$ & 2,07 & $1,2-3,7$ & 9,33 & 4,5-19 & 3,65 & $1,5-9,0$ \\
\hline
\end{tabular}

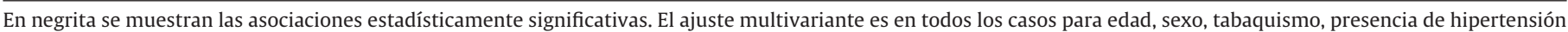

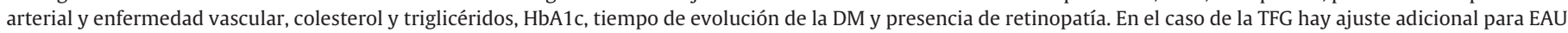
y en el caso de la EAU hay ajuste adicional para la TFG.

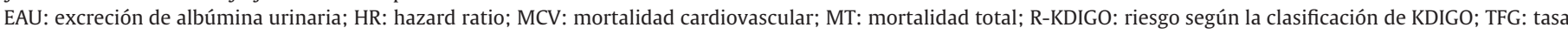
de filtración glomerular. 
a más largo plazo (18 años) de esta misma cohorte confirmó la necesidad de considerar la DM un equivalente coronario ${ }^{11}$. Por el contrario, un estudio ${ }^{12}$ de las redes GEDAPS y REGICOR en nuestro país comunicó un riesgo de enfermedad coronaria inferior en DM tipo 2 sin historia de infarto que en sujetos con un infarto previo ( $\mathrm{HR}=0,43$; IC 95\% 0,36-0,51), con una tasa de MCV en los pacientes con DM $(4,4 / 1000)$ muy inferior a la descrita en el estudio de Haffner et al. ${ }^{1}$ (25/1000), a pesar de que la edad de los pacientes era similar. En un amplio estudio epidemiológico ${ }^{13}$ de referencia realizado por el Emerging Risk Factors Collaboration, con inclusión de 820900 participantes, la presencia de DM duplicó aproximadamente el riesgo de MT y MCV, con tasas de MCV de 13/1000 en varones y 11/1000 en mujeres con DM. En nuestro estudio la tasa de MCV global fue de 14,1/1000, similar por tanto a la descrita en el estudio anterior.

Aunque según los datos anteriores en promedio el paciente con DM puede considerarse de muy alto riesgo vascular, la justificación de tasas tan dispares entre los diferentes estudios puede estar en el perfil de pacientes incluidos. Debido a la elevada prevalencia de la DM en nuestro país ${ }^{14}$, estimada en un $13,8 \%$, se hace necesario discriminar el riesgo vascular de los pacientes para decidir la intensidad del tratamiento de los factores de riesgo, especialmente lipídicos. En una revisión reciente se llegó a la conclusión de que, en pacientes con DM tipo 2, los modelos predictivos alcanzan una discriminación tan solo moderada, por lo que es difícil predecir la aparición de episodios vasculares ${ }^{15}$. Por ello el reto es identificar con precisión aquellos pacientes con DM que se hallan en el extremo más desfavorable del espectro del riesgo vascular.

En una revisión ${ }^{4}$ de ensayos clínicos que incluyesen al menos 1000 pacientes con DM tipo 2 y que evaluasen resultados de MT, se demostró una variabilidad en las tasas de MT entre 2,8 y 84,2/1000 pacientes-año; un hallazgo consistente fue que las tasas más elevadas se producían en los ensayos que incluían pacientes con ERC. Del mismo modo en nuestro estudio hubo una amplia oscilación en las tasas de MT $(26,5-109,8)$ y de $\operatorname{MCV}(8,1-57,5)$ según la clasificación de riesgo propuesta por las guías KDIGO. Dicha clasificación demostró criterios de validez para estratificar el riesgo de MT y MCV de los pacientes: asociación con un gradiente dosis-respuesta (incremento significativo de riesgo a medida que se avanzaba de categoría) y efecto significativo en análisis multivariante con ajuste para potenciales factores de confusión, incluida la principal complicación microangiopática, la presencia de retinopatía. Otros factores predictivos de MT y/o MCV, además de la edad, fueron similares a los descritos en la literatura: presencia de enfermedad vascular inicial $^{1,16}$, presencia de retinopatía ${ }^{17}$ y tabaquismo activo ${ }^{18}$. Es de destacar además que la clasificación funcionó mejor, en el límite de la significación estadística, para predecir MT en el subgrupo de pacientes sin macroangiopatía inicial; este hecho refuerza su utilidad, ya que es en los pacientes sin macroangiopatía en los que más necesaria es una estratificación del riesgo vascular. La inclusión de la clasificación KDIGO permitió identificar correctamente a un $11,6 \%$ de los pacientes sin ECV inicial como de riesgo extremo, con un riesgo de MCV similar al de los pacientes con ECV establecida ${ }^{6}$. La reciente incorporación terapéutica de los inhibidores de PCSK-9 para el tratamiento de la dislipemia hace necesaria la identificación de estos pacientes con mayor riesgo absoluto, en los que su administración puede resultar coste-efectiva ${ }^{19}$.

La utilidad de la clasificación de riesgo KDIGO viene derivada de recoger información de la TFG y de la EAU de los pacientes. Al igual que en población general, en los paciente con DM hay un incremento progresivo de riesgo de MT y MCV a medida que desciende la TFG y se incrementa la EAU ${ }^{20}$. En un estudio de seguimiento durante 4 años de 42761 pacientes con DM incluidos en la base $\mathrm{KEEP}^{21}$, las tasas de mortalidad oscilaron entre 3,1 (pacientes con TFG $>105 \mathrm{ml} / \mathrm{min}$ por $1,73 \mathrm{~m}^{2}$ y normoalbuminuria) y $73,7 / 1000$ (pacientes con TFG $<30 \mathrm{ml} / \mathrm{min}$ por $1,73 \mathrm{~m}^{2}$ y macroalbuminuria); el término de interacción entre TFG y EAU fue significativo, indicando una amplificación del riesgo ante la presencia simultánea de una alteración de ambos parámetros. En un análisis previo de nuestros datos ${ }^{22}$, comprobamos que tanto la TFG como la EAU eran factores predictivos independientes de MT.

Por todo ello consideramos que la clasificación de riesgo KDIGO puede ser útil para una evaluación rápida del riesgo vascular del paciente en una variable combinada. Hay que tener en cuenta además que la evolución en el tiempo tanto de la $\mathrm{TFG}^{23}$ como de la EAU $^{24}$ ofrecen información pronóstica adicional, por lo que consideramos que debe realizarse evaluación periódica del estrato en el que se encuentra el paciente.

La mayor fortaleza de nuestro trabajo es el haber podido mantener un seguimiento durante más de 10 años de los pacientes, con escasas pérdidas, lo que permite una estimación fiable de las tasas de episodios durante este plazo de tiempo y refuerza la validez interna. En cuanto a las debilidades a destacar hay que señalar el limitado número de sujetos con un número reducido de episodios en algunas categorías, lo que hace más imprecisas las estimaciones. Por otro lado se trata de pacientes con DM tipo 2 seleccionados en consultas externas hospitalarias, con una edad media de 65 años y un tiempo de evolución medio de la DM de más de 10 años, lo que limita la validez externa de los resultados. Asimismo solo tuvimos datos de la clasificación KDIGO en el momento inicial, por lo que no pudimos evaluar el impacto pronóstico del cambio de categoría durante la evolución.

En nuestro trabajo hemos demostrado que una clasificación de riesgo en 4 categorías, recogida en las guías KDIGO 2012, es válida para predecir mortalidad total y mortalidad cardiovascular en pacientes con diabetes tipo 2. La utilización de esta clasificación puede ser útil para una estratificación rápida del riesgo de mortalidad cardiovascular de los pacientes, para establecer los objetivos de control de factores de riesgo a alcanzar y para realizar una evaluación de coste-efectividad de las nuevas intervenciones terapéuticas.

\section{Conflicto de intereses}

Los autores declaran no tener ningún conflicto de intereses.

\section{Bibliografía}

1. Haffner M, Lehto S, Ronnemaa T, Pyorala K, Laakso M. Mortality from coronary heart disease in subjects with type 2 diabetes and in nondiabetic subjects with and without prior myocardial infarction. N Engl J Med. 1998;339:229-34.

2. Bonaca MP, Gutierrez JA, Cannon C, Giugliano R, Blazing M, Park JG, et al. Polyvascular disease, type 2 diabetes, and long-term vascular risk: a secondary analysis of the IMPOVE-IT trial. Lancet Diabetes Endocrinol. 2018;6:934-43.

3. Chronic Kidney Disease Prognosis, Consortium. Association of estimated glomerular filtration rate and albuminuria with all-cause and cardiovascular mortality in general population cohorts: a collaborative, meta-analysis. Lancet. 2010;375:2073-81

4. Barkoudah E, Skali H, Uno H, Solomon SD, Pfeffer MA. Mortality rates in trials of subjects with type 2 diabetes. J Am Heart Assoc. 2012;1:8-15.

5. Piepoli MF, Hoes AW, Agewall S, Albus C, Brotons C, Catapano AL, et al. 2016 European Guidelines on cardiovascular disease prevention in clinical practice. Eur J Prev Cardiol. 2016;23. NP1-NP96.

6. Garber AJ, Abrahamson MJ, Barzilay JI, Blonde L, Bloomgarden ZT, Bush MA, et al. Consensus statement by the American Association of Clinical Endocrinologists and American College of Endocrinology on the comprehensive type 2 diabetes management algorithm - 2018 Exectutive Summary. Endocrine Practice. 2018;24:91-120

7. Gorostidi M, Santamaría R, Alcázar R, Fernández-Fresnedo G, Galcerán JM, Goicoechea M, et al. Spanish Society of Nephrology document on KDIGO guidelines for the assessment and treatment of chronic kidney disease. Nefrologia. 2014;34:302-16

8. Gimeno-Orna JA, Boned-Juliani B, Lou-Arnal LM, Castro-Alonso FJ. Mortality in a cohort of patients with type 2 diabetes mellitus of Alcañiz area Spain. An Med Interna. 2002;19:336-40

9. Gimeno-Orna JA, Castro-Alonso FJ, Sánchez-Vañó R, Latre-Rebled B, Lou-Arnal LM, Molinero-Herguedas E. Diabetic retinopathy and mortality in type 2 diabetic patients. Med Clin (Barc). 2006;126:686-9.

10. World Health Organization Study Group on Diabetes Mellitus. Technical report series 727. 1985: WHO, Geneva. 
11. Juutilainen A, Lehto S, Ronnemaa T, Pyorala K, Laakso M. Type 2 diabetes as a "coronary heart disease equivalent". Diabetes Care. 2005;28:2901-7.

12. Cano JF, Baena-Diez J, Franch J, Vila J, Tello S, Sala J, et al. Longterm cardiovascular risk in type 2 diabetic compared with nondiabetic first acute myocardial infarction patients. Diabetes Care. 2010;33: 2004-9.

13. The Emerging Risk Factors Collaboration. Diabetes mellitus, fasting glucose and risk of cause-specific death. N Engl J Med 2011; 364: 829-41.

14. Soriguer F, Goday A, Bosch-Comas A, Bordiú E, Calle-Pascual A, Carmena R, et al. Prevalence of diabetes mellitus and impaired glucose regulation in Spain: the Di@bete.es Study. Diabetologia. 2012;55:88-93.

15. Allan GM, Nouri F, Korownyk C, Kolber MR, Vandermeer B, McCormack J. Agreement among cardiovascular disease risk calculators. Circulation. 2013;127:1948-56.

16. Giorda CB, Avogaro A, Maggini M, Lombardo F, Mannucci E, Turco S, et al. Recurrence of cardiovascular events in patients with type 2 diabetes. Diabetes Care. 2008;31:2154-9.

17. Kramer CK, Rodrigues TC, Canani LH, Gross JL, Azevedo MJ. Diabetic retinopathy predicts all-cause mortality and cardiovascular events in both type 1 and 2 diabetes. Diabetes Care. 2011;34:1238-44.

18. Clair C, Rigotti NA, Porneala B, Fox CS, DÁgostino RB, Pencina MJ, et al. Association of smoking cessation and weight change with cardiovascular disease among adults with and without diabetes. JAMA. 2013;309:1014-21.
19. Landmesser U, Chapman MJ, Stock JK, Amarenco P, Belch JJ, Borén J, et al. 2017 Update of ESC/EAS Task Force on practical clinical guidance for proprotein convertase subtilisin/kesin type 9 inhibition in patientes with atherosclerotic cardiovascular disease or in familial hypercholesterolaemia. Eur Heart J. 2017, http://dx.doi.org/10.1093/eurheartj/ehx549.

20. Fox CS, Matsushita K, Woodward M, Bilo HJG, Chalmers J, Heerspink HJL, et al. Associations of kidney disease measures with mortality and end-stage renal disease in individuals with and without diabetes: a meta-analysis. Lancet. 2012;380:1662-73.

21. Amin AP, Whaley-Connell AT, Li S, Chen SC, McCullough PA, Kosiborod MN. The synergistic relationship between estimated GFR and microalbuminuria in predicting long-term progression to ERSD or death in patients with diabetes: results from the Kidney Early Evaluation Program (KEEP). Am J Kidney Dis. 2013;61 suppl 2. S12-S23.

22. Gimeno-Orna JA, Blasco-Lamarca Y, Campos-Gutierrez B, Molinero-Herguedas E, Lou-Arnal LM, García-García B. Riesgo de mortalidad asociado a enfermedad renal crónica en pacientes con diabetes tipo 2 durante un seguimiento de 13 años. Nefrología. 2015;35:487-92.

23. Coresh J, Turin TC, Matsushita K, Sang Y, Ballew SH, Appel LJ, et al. Decline in estimated glomerular filtration rate and subsequent risk of end-stage renal disease and mortality. JAMA. 2014;311:2518-31.

24. Bakris GL, Molitch M. Microalbuminuria as a risk predictor in diabetes: the continuing saga. Diabetes Care. 2014;37:867-75. 